Check for updates

Cite this: RSC Adv., 2017, 7, 37938

Received 16th May 2017

Accepted 19th July 2017

DOI: 10.1039/c7ra05505f

rsc.li/rsc-advances

\title{
One-pot synthesis of hierarchical concave tetrapod Pd nanocrystals and their electrocatalytic properties $\dagger$
}

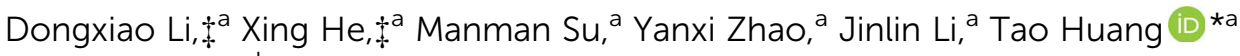 \\ and Hanfan Liu ${ }^{\text {ab }}$
}

Well-defined Pd nanocrystals with both hierarchical pods and concave centers were synthesized by reducing $\mathrm{Pd}(\mathrm{acac})_{2}$ with $\mathrm{CO}$ in the presence of polyvinylpyrrolidone and oleylamine in DMF. Oleylamine was responsible for the generation of the hierarchical concave tetrapod Pd nanostructures. The asprepared novel Pd nanocrystals demonstrated highly enhanced electrocatalytic activity.

As one of the most studied platinum group metallic nanomaterials, Pd nanocrystals with well-defined morphologies have continually received much attention for years because of their fantastic properties and their widespread applications in catalysis. For instance, they have been widely used as catalysts in organic reactions, hydrogenation, fuel cell reactions, as well as the low-temperature catalytic reduction of engine exhaust species. In order to improve their catalytic efficiency, many Pd nanoparticles with different shapes such as nanowires, ${ }^{1}$ nanorods, ${ }^{2}$ nanosheets, ${ }^{3}$ nanocubes, ${ }^{4}$ octahedra, ${ }^{5}$ icosahedra, ${ }^{6}$ and tetrahexahedra ${ }^{7}$ have been successfully created by various strategies. In recent years, concave, ${ }^{8}$ branched $^{9}$ or multipod $^{10}$ nanostructures, which are different from those enclosed by flat and convex surfaces, have aroused extraordinary interest due to the presence of more unsaturated atoms, higher surface energy (and thus more active sites resulting from their special shapes), sharp corners, as well as more atomic steps and kinks. Although several processes for the synthesis of Pd nanocrystals with concave surfaces, multipod or hierarchical arms have been developed, it is still a great challenge to create further novel Pd nanostructures, such as hierarchical multipods with concave surfaces.

Herein, we demonstrate a facile one-pot synthesis of hierarchical concave tetrapod Pd nanocrystals, a unique nanostructure with both arthrogenous pods and concave centers, which are quite different those reported multipod nanostructures such as Pd tetrapod nanocrystals, three-dimensional

\footnotetext{
${ }^{a}$ Key Laboratory of Catalysis and Material Science of the State Ethnic Affairs Commission, Ministry of Education, College of Chemistry and Material Science, South-Central University for Nationalities, Wuhan 430074, Hubei Province, China. E-mail: huangt208@163.com

${ }^{b}$ Institute of Chemistry, Chinese Academy of Science, Beijing 100080, China

$\dagger$ Electronic supplementary information (ESI) available. See DOI: 10.1039/c7ra05505f

$\$ \mathrm{D}$. Li and X. He contributed equally.
}

highly branched palladium tetrapods, and hierarchical tetrapod Pd nanocrystals. Oleylamine (OAm) was critical to the formation of the hierarchical concave tetrapod Pd nanocrystals. The asprepared Pd nanocrystals demonstrated highly enhanced electrocatalytic activity.

Fig. 1 shows the typical TEM images of the as-prepared Pd nanocrystals. As can be seen, nearly all of the projections of the Pd nanoparticles present as tripod-like profiles under TEM (Fig. 1a). The average apex-to-apex dimension was about $175 \mathrm{~nm}$. In fact, the dominant morphological characteristic of the as-prepared Pd particles was tetrapod-shaped nanostructures and each one should appears four hierarchical pods with tetrahedral distribution since the preferential orientation tends to lie flat onto the substrate by using three of their four vertexes. Furthermore, Fig. 1b shows the vertical projection of an individual tetrapod Pd particle along a vertex, in which one of the four pods was oriented towards the observing direction, while Fig. 1c exhibits the rear projection along the bottom of one tetrapod. As can be seen, both of them display a clear hierarchical feature for each pod. It is particularly noteworthy that the bottom projection demonstrates a concave feature in which a trigonal pyramid cavity was excavated at the central part (Fig. 1c). Obviously, the cut-out pyramid occurs at each bottom centre of a tetrapod Pd nanocrystal.

This morphological feature was confirmed further by SEM measurements. As can be seen, the metameres embedded mutually one after another for each arthrogenous pod (Fig. 1d and e) as well as the concave center (Fig. If and S1, ESI $\dagger$ ) are present clearly. Accordingly, the as-prepared Pd nanocrystal with both hierarchical and concave feature is quite different from those just merely hierarchical tetrapods or concave tetrahedral nanocrystals.

Coupled HRTEM and selected-area electron diffraction (SAED) measurements on the partial hierarchical pod of an individual Pd nanocrystal determined their lattice parameters 


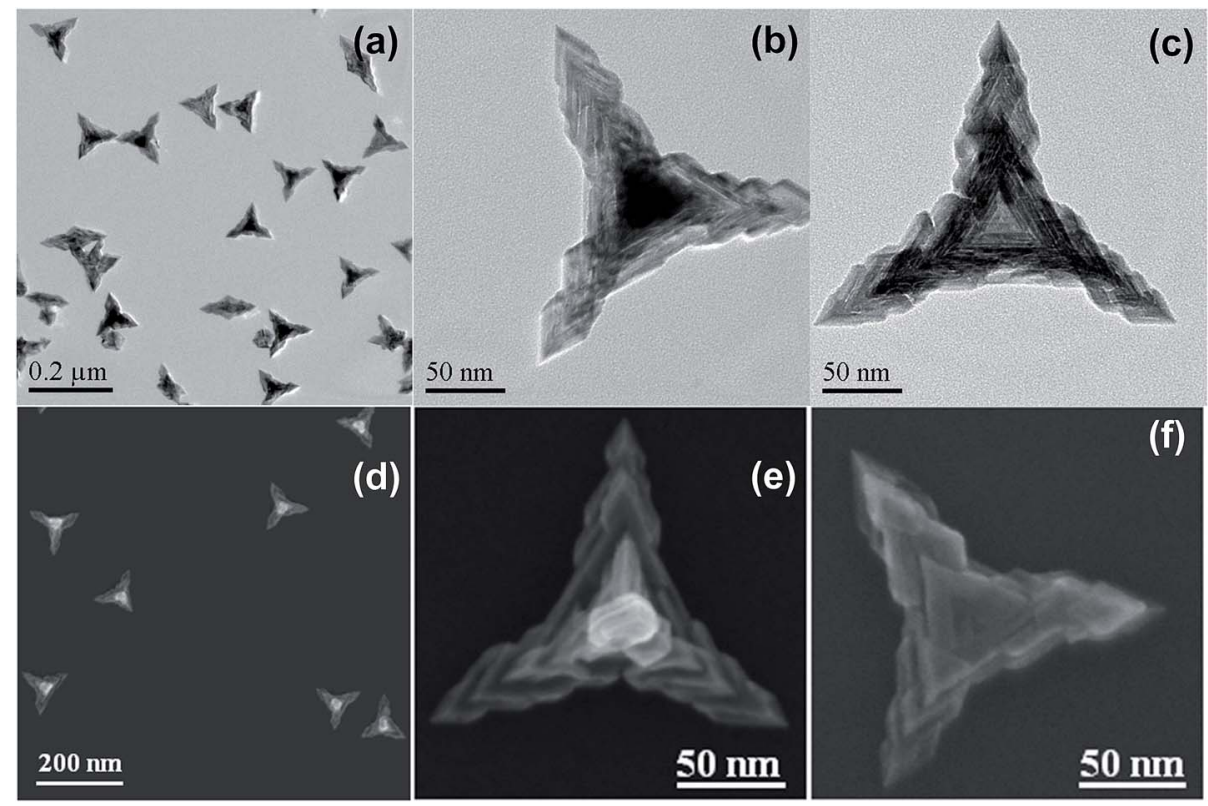

Fig. 1 (a) TEM image of the hierarchical concave tetrahedral Pd nanocrystals; (b) and (c) TEM images of an individual Pd nanocrystal observed from a vertex and a bottom, respectively; (d) SEM image; (e) and (f) SEM images of an individual Pd nanocrystal corresponding to (b) and (c), respectively.

and the zone axis. HRTEM images show lattice fringes with an interplanar spacing of $0.236 \mathrm{~nm}$ (Fig. $2 \mathrm{a}$ and b), corresponding to the (111) facets of face-centered cubic (fcc) Pd. The SAED pattern was indexed to the [111] zone axis and revealed their single-crystalline structure (Fig. 2c). These observations implied that the due to multi-generation overgrowth of the hierarchical concave Pd tetrapod, which originated from the vertices of the concave tetrahedral Pd core, may be ascribed to vertex-selective atomic deposition along the $<111>$ axis directions owing to the highest surface energy at the vertices.

The exposed $\{111\}$ surfaces were further supported by CO stripping voltammetry. As shown in Fig. S2a (ESI $\dagger$ ), only one peak of CO electro-oxidation $\left(\mathrm{CO}_{\mathrm{ox}}\right)$ at $0.966 \mathrm{~V}$ (versus SCE) was observed for the freshly prepared hierarchical concave tetrapod Pd nanocrystals in $0.1 \mathrm{M} \mathrm{H}_{2} \mathrm{SO}_{4}$, which can be assigned to $\mathrm{CO}$ stripping on Pd $\{111\}$ surfaces, implying the existence of preferential (111) planes for the final products. Followed by a second scan, no $\mathrm{CO}_{\text {ox }}$ peak was observed (Fig. S2b, ESI†).

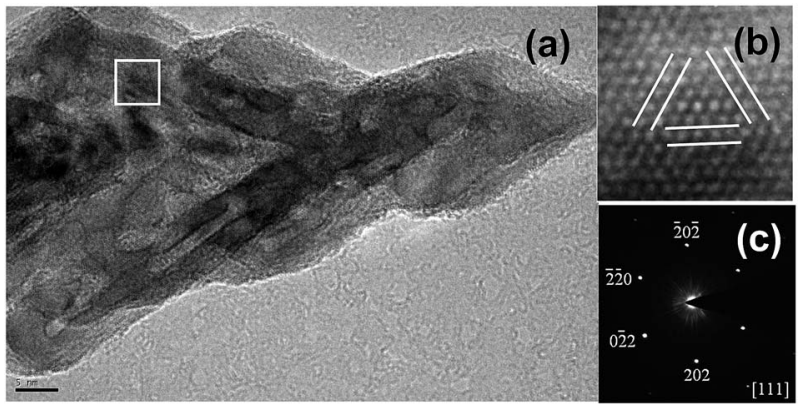

Fig. 2 (a) High magnified TEM image of one pod. (b) HRTEM image; (c) SAED pattern.
Subsequently, the $\mathrm{CO}_{\text {ox }}$ peak appeared again at $0.960 \mathrm{~V}$ (Fig. S2c, ESI $\dagger$ ) for the CO-stripped Pd-modified electrode after CO readsorption, which was still ascribed to CO stripping on Pd $\{111\}$ surfaces. These measurements showed that $\mathrm{CO}$ was adsorbed preferentially on (111) planes in the growth of hierarchical concave tetrapod Pd nanocrystals. On the other hand, it also shows that the formation of this Pd nanostructure should be ascribed to the selective adsorption of $\mathrm{CO}$ on (111) planes.

Moreover, the powder X-ray diffraction (XRD) pattern of the hierarchical concave tetrapod Pd nanocrystals shows five peaks at $40.02^{\circ}, 46.53^{\circ}, 67.79^{\circ}, 81.88^{\circ}$ and $86.16^{\circ}$ (Fig. 3), corresponding to the (111), (200), (220), (311) and (222) lattice planes, respectively, which matched well with the standard metallic Pd diffractions (JCPDS card no. 05-0681), confirming the high purity and high crystallinity of the as-prepared Pd nanocrystals. It is noted that these Pd nanocrystals exhibit a very strong (111)

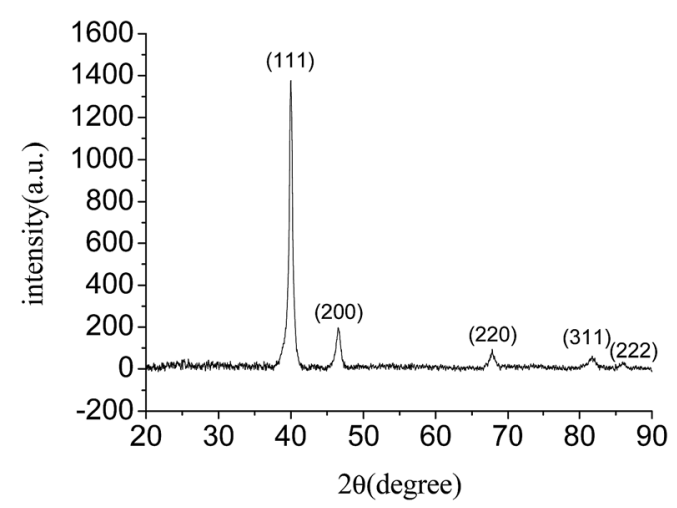

Fig. 3 XRD pattern of the hierarchical concave tetrahedral $\mathrm{Pd}$ nanocrystals. 
diffraction peak. The intensity ratio of the (111) to (200) peaks is 7.02 , which is much higher than that of the bulk values of a conventional Pd powder sample (2.38). These observations indicated that the preferential orientation of the hierarchical concave tetrapod Pd nanocrystals was (111) planes, which was consistent with the HRTEM observation. In addition, XPS measurement demonstrated that the binding energy of $\mathrm{Pd} 3 \mathrm{~d}_{5 / 2}$ and $\mathrm{Pd} 3 \mathrm{~d}_{3 / 2}$ were 335.15 and $340.40 \mathrm{eV}$ (Fig. S3, ESI $\dagger$ ), respectively, showing in good agreement with the corresponding reference values of $\mathrm{Pd}^{0}$ (335.10 and $\left.340.36 \mathrm{eV}\right) .{ }^{11}$

It was found that OAm was critical to the growth of the hierarchical concave tetrapod Pd nanocrystals. As shown in Fig. 4a and S4a (ESI $\dagger$ ), the absence of OAm led to producing tetrahedral Pd nanoparticles with a mean apex-to-apex size of $45 \mathrm{~nm}$. When the amount of OAm was $0.1 \mathrm{~mL}$, concave tetrahedral nanocrystals with an average dimension of $50 \mathrm{~nm}$ were obtained (Fig. 4b and S4b ESI $\dagger$ ). With further increasing the amount of OAm from 0.3 to $0.5 \mathrm{~mL}$, vertex-selective deposition along the vertexes of concave tetrahedron occurred and their average dimension was 85 and $135 \mathrm{~nm}$, respectively (Fig. 4c and d), though the hierarchical feature for each pod was not obvious. These observations suggested that both the formation of concave centres and the growth of hierarchical pods are significantly dependent upon the amount of OAm.

Furthermore, the effect of different amines was investigated. Only agglomerated sheets were observed by using ethylamine instead of OAm (Fig. 5a), while concave tetrahedral Pd nanoparticles were preferred by using butylamine instead (Fig. 5b). Nevertheless, similar hierarchical concave tetrapod Pd nanocrystals were obtained by using dodecylamine instead of OAm, as shown in Fig. 5c and S5 (ESI $\dagger$ ), except for their slightly smaller size than that of OAm case (Fig. 5d). These results

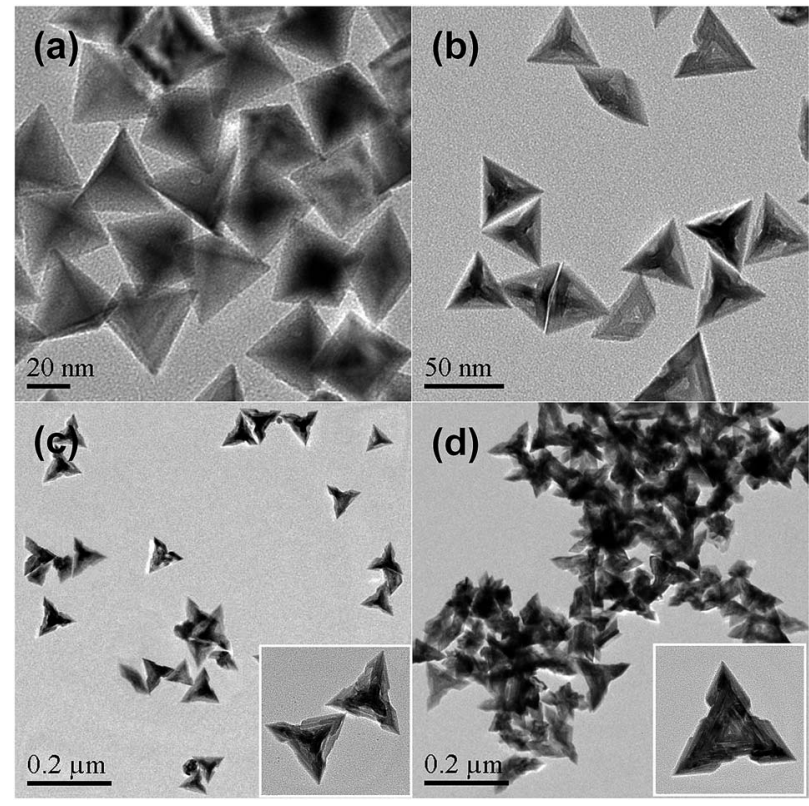

Fig. 4 TEM images of the Pd nanocrystals prepared by using different amount of OAm. (a) $0 \mathrm{~mL} \mathrm{OAm;} \mathrm{(b)} 0.1 \mathrm{~mL}$; (c) $0.3 \mathrm{~mL} \mathrm{OAm;} \mathrm{(d)} 0.5 \mathrm{~mL}$ OAm. The insets in (c) and (d) are the corresponding TEM images of an individual Pd nanocrystal, respectively.

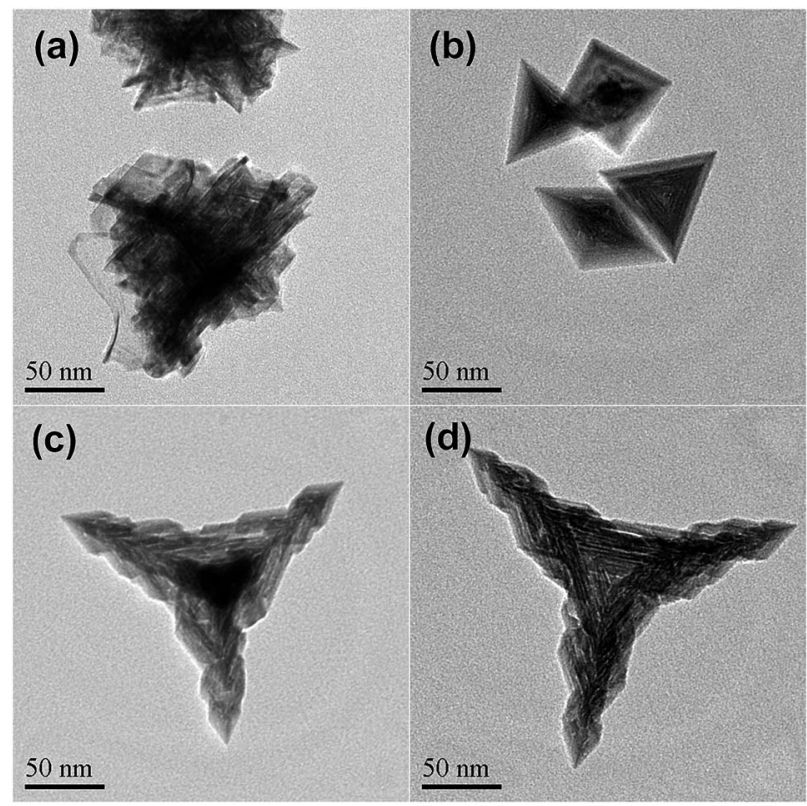

Fig. 5 TEM images of the hierarchical concave tetrahedral Pd nanocrystals prepared with different amines. (a) Ethylamine; (b) butylamine; (c) dodecylamine; (d) oleylamine. The inset in (c) is the corresponding TEM image of an individual Pd nanocrystal.

implied that the generation of hierarchical concave tetrapod Pd nanocrystals was dependent upon the alkyl chain length of amine, which may resulted in different coverage due to the adsorption of amines on surfaces of Pd nanoparticles. The coverage caused by OAm was better for the formation of hierarchical tetrapod nanostructure.

In addition, the effect of the flow rate of $\mathrm{CO}$ was also investigated. As shown in Fig. 4a, CO acted as one of the shapecontrolling agents. However, no Pd particle was obtained without using $\mathrm{CO}$ under the same reaction conditions, indicating that $\mathrm{CO}$ also acted as the main reducing agent. When $\mathrm{CO}$ was bubbled with a lower rate of 0.05 or $0.1 \mathrm{~mL} \mathrm{sec}^{-1}$, hierarchical concave tetrapod Pd nanocrystals were observed all in these cases, as shown in Fig. S6 (ESI†). These observations suggested that a lower CO flow rate had little effect on the morphologies of the final $\mathrm{Pd}$ nanostructures except their uniformities.

The effect of reaction temperature on the morphologies of $\mathrm{Pd}$ nanoparticles was also investigated. Keeping other conditions unchanged, hierarchical growth characteristics with a continuous accumulation of concave tetrahedron along the vertexes one after another were observed obviously at a relatively lower temperature $\left(80^{\circ} \mathrm{C}\right)$ except for bigger gaps between metameres (Fig. S7a, ESI $\dagger$ ). When the temperature increased to $100{ }^{\circ} \mathrm{C}$, hierarchical concave tetrapod structures with fewer metameres were formed (Fig. S7b, ESI $\dagger$ ). At $120{ }^{\circ} \mathrm{C}$, regular hierarchical concave tetrapod Pd nanoparticles were generated (Fig. S7c, $\mathrm{ESI}^{\dagger}$ ). Nevertheless, inhomogeneous hierarchical concave tetrapods were obtained at a higher temperature $\left(160{ }^{\circ} \mathrm{C}\right)$ (Fig. S7d, ESI $\dagger$ ). These results demonstrated that vertex-selective Pd atomic deposition was independent upon the temperature except the extent of metamere growth. Of course, appropriate 
temperature is favourable for the formation of uniform and well-defined hierarchical concave tetrapod Pd nanocrystals. The optimum temperature should be 120 to $140{ }^{\circ} \mathrm{C}$.

Combined with the influences of $\mathrm{CO}, \mathrm{OAm}$ and other amines as above results, they suggested that the selective adsorption of CO molecules on the (111) planes was favourable for the generation of tetrahedral Pd nanoparticles as shown in the case of OAm absent (Fig. 4a), while the presence of a small amount of OAm or a short-chain amine was beneficial for the formation of concave tetrahedral Pd due to their selective adsorption on the centre of each triangular face. Moreover, the use of sufficient amount of OAm or other long-chain amine prompted vertexselective Pd atomic deposition due to confinement effect of amines. As a result, hierarchical concave tetrapod nanostructures were formed because the long alkyl chain coating on the surface of Pd nanoparticles blocked the facet-selective atomic deposition. Accordingly, OAm played an important role in the formation of the hierarchical concave tetrapod Pd nanocrystals. The selective adsorption of an appropriate amount of OAm on the surfaces of Pd nanoparticles led to vertex-selective Pd atomic deposition, resulting in the formation of hierarchical concave tetrapod Pd nanostructures. The morphological evolution developed from tetrahedral to hierarchical concave tetrapod nanostructure can be illustrated as Fig. S8 (ESI $\dagger$ ) with the increase of the amount of OAm.

The catalytic performances of the hierarchical concave tetrapod Pd nanocrystals were investigated preliminarily by electrochemical oxidation of formic acid. Fig. 6 plots the cyclic voltammetry (CV) curves for the electrocatalytic oxidation of formic acid. The oxidation peak current density for the hierarchical concave tetrapod Pd nanocrystals was measured to be $4.94 \mathrm{~mA} \mathrm{~cm}^{-2}$ at $0.251 \mathrm{~V}$, while $1.70 \mathrm{~mA} \mathrm{~cm}{ }^{-2}$ for Pd black at $0.244 \mathrm{~V}$ and $1.40 \mathrm{~mA} \mathrm{~cm}^{-2}$ for $\mathrm{Pd} / \mathrm{C}$ at $0.238 \mathrm{~V}$, respectively. The activity of the as-prepared hierarchical concave tetrapod Pd nanocrystals, though holding a large mean size, was 2.9 and 3.5 times that of Pd black and Pd/C, respectively, demonstrating an outstanding electrocatalytic activity. The enhancement of their catalytic activity may be ascribed to their unique structures on

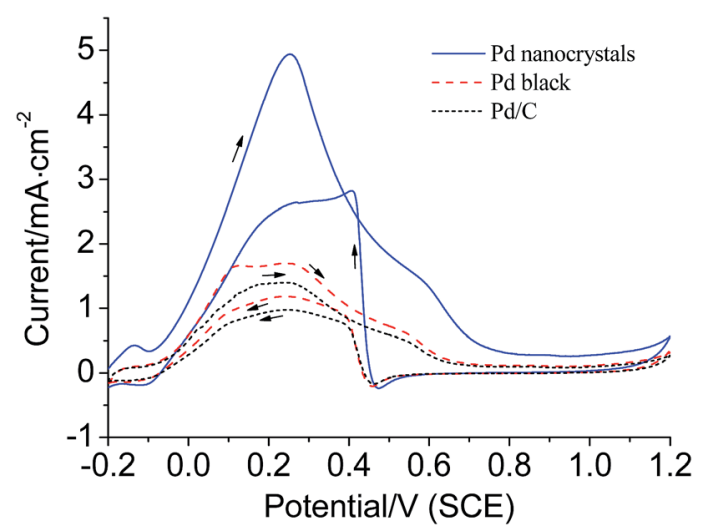

Fig. 6 CV curves for electro-oxidation of formic acid by the hierarchical concave tetrapod $\mathrm{Pd}$ nanocrystals and $\mathrm{Pd}$ black recorded in $0.5 \mathrm{M} \mathrm{H}_{2} \mathrm{SO}_{4}+0.5 \mathrm{M} \mathrm{HCOOH}$ solution at a scan rate of $50 \mathrm{mV} \mathrm{s}^{-1}$ between -0.2 and $1.2 \mathrm{~V}$. account of their large surface areas, multiple high-angle edges, and sharp corners, though their main exposed planes are low index (111) facets.

In summary, a facile one-pot synthetic method of hierarchical concave tetrapod Pd nanocrystals was developed by using $\operatorname{Pd}(\mathrm{acac})_{2}$ as a precursor, $\mathrm{CO}$ as a reducing agent, PVP as a stabilizer, OAm as a shape-controlling agent in DMF at $140{ }^{\circ} \mathrm{C}$ for $3 \mathrm{~h}$. The existence of OAm was critical to the generation of the hierarchical concave tetrapod Pd nanostructures. Due to confinement effect resulting from the adsorption of OAm, vertex-selective Pd atomic deposition at the vertexes of concave tetrahedral nucleus along <111> direction resulted in the formation of hierarchical concave tetrapod nanocrystals. The electrocatalytic activity of the as-prepared hierarchical concave tetrapod Pd nanocrystals was significantly enhanced.

\section{Acknowledgements}

This work was supported by the National Nature Science Foundation of China (Grant 21273289) and the Fundamental Research Funds for the Central Universities (CZW15039).

\section{Notes and references}

1 X. Q. Huang and N. F. Zheng, J. Am. Chem. Soc., 2009, 131, 4602-4603.

2 (a) Y. J. Xiong, H. G. Cai, B. J. Wdey, J. G. Wang, M. J. Kim and Y. N. Xia, J. Am. Chem. Soc., 2007, 129, 3665-3675; (b) Y.-H. Chen, H.-H. Hung and M. H. Huang, J. Am. Chem. Soc., 2009, 131, 9114-9121.

3 (a) X. Huang, S. Tang, X. Mu, Y. Dai, G. Chen, Z. Zhou, F. Ruan, Z. Yang and N. Zheng, Nat. Nanotechnol., 2011, 6, 28-32; (b) H. Li, G. Chen, H. Yang, X. Wang, J. Liang, P. Liu, M. Chen and N. Zheng, Angew. Chem., Int. Ed., 2013, 52, 8368-8372; (c) X. Yin, X. Liu, Y. T. Pan, K. A. Walsh and H. Yang, Nano Lett., 2014, 14, 7188-7194.

4 (a) J. T. Ren. and R. D. Tilley, J. Am. Chem. Soc., 2007, 129, 3287-3291; (b) Y. Yu, Y. Zhao, T. Huang and H. Liu, Mater. Res. Bull., 2010, 45, 159-164.

5 M. Liu, Y. Zheng, L. Zhang, L. Guo and Y. Xia, J. Am. Chem. Soc., 2013, 135, 11752-11755.

6 (a) Y. Xiong, J. M. McLellan, Y. Yin and Y. Xia, Angew. Chem., Int. Ed., 2007, 46, 790-794; (b) Y. Chen, B. He, T. Huang and H. Liu, Colloids Surf., A, 2009, 348, 145-150; (c) Y. Yu, Y. Zhao, T. Huang and H. Liu, Pure Appl. Chem., 2009, 81, 2377-2385.

7 N. Tian, Z.-Y. Zhou, N.-F. Yu, L. Y. Wang and S.-G. Sun, J. Am. Chem. Soc., 2010, 132, 7580-7581.

8 (a) X. Huang, S. Tang, H. Zhang, Z. Zhou and N. Zheng, J. Am. Chem. Soc., 2009, 131, 13916-13917; (b) H. Zhu, Q. Chi, Y. Zhao, C. Li, H. Tang, J. Li, T. Huang and H. Liu, Mater. Res. Bull., 2012, 47, 3637-3643; (c) M. Jin, H. Zhang, Z. Xie and Y. Xia, Angew. Chem., Int. Ed., 2011, 50, 7850-7854.

9 (a) J. Watt, N. Young, S. Haigh, A. Kirkland and R. D. Tilley, Adv. Mater., 2009, 21, 2288-2293; (b) R. Zhao, G. Fu, T. Zhou, Y. Chen, X. Zhu, Y. Tang and T. Lu, Nanoscale, 2014, 6, 27762781; (c) H. Zhu, G. Li, X. Lv, Y. Zhao, T. Huang, H. Liu and J. Li, RSC Adv., 2014, 4, 6535-6539. 
10 (a) Y. Dai, X. Mu, Y. Tan, K. Lin, Z. Yang, N. Zheng and G. Fu, J. Am. Chem. Soc., 2012, 134, 7073-7080; (b) H. Zhu, G. Li, Q. Chi, Y. Zhao, H. Liu, J. Li and T. Huang, CrystEngComm, 2012, 14, 1531-1533.
11 C. D. Wagner, W. M. Riggs, L. E. Davis, J. F. Moulder and B. E. Muilenberg, Handbook of X-ray Photoelectron Spectroscopy, Perkin-Elmer, Physical Electronics Division, Eden Prairie, 1979. 